\title{
Real continuation from the complex quadratic family: Fixed point bifurcation sets
}

Bruce B. Peckham

Department of Mathematics and Statistics

University of Minnesota, Duluth

Duluth, Minnesota 55812

bpeckham@d.umn.edu

\author{
James Montaldi \\ Institut NonLinéaire de Nice \\ Université de Nice - Sophia Antipolis \\ 06560 Valbonne, France \\ montaldi@inln.cnrs.fr
}

June 14, 1999

\begin{abstract}
This paper is primarily a numerical study of the fixed-point bifurcation loci - saddle-node, period-doubling and Hopf bifurcations - present in the family:

$$
z \rightarrow f_{(C, A)}(z, \bar{z}) \equiv z+z^{2}+C+A \bar{z}
$$

where $z$ is a complex dynamic (phase) variable, $\bar{z}$ its complex conjugate, and $C$ and $A$ are complex parameters. We treat the parameter $C$ as a primary parameter and $A$ as a secondary parameter, asking how the bifurcation loci projected to the $C$ plane change as the auxiliary parameter $A$ is varied. For $A=0$, the resulting two-real-parameter family is a familiar one-complex-parameter quadratic family, and the local fixed-point bifurcation locus is the main cardioid of the Mandlebrot set. For $A \neq 0$, the resulting two-real-parameter families are not complex analytic, but are still analytic (quadratic) when viewed as a map of $\mathcal{R}^{2}$. Saddle-node and period-doubling loci evolve from points on the main cardioid for $A=0$ into closed curves for $A \neq 0$. As $A$ is varied further from 0 in the complex plane, the three sets interact in a variety of interesting ways.

More generally, we discuss bifurcations of families of maps with some parameters designated as primary and the rest as auxiliary. The auxiliary parameter space is then divided into equivalence classes with respect to a specified set of bifurcation loci. This equivalence is defined by the existence of a diffeomorphism of corresponding primary parameter spaces which preserves the specified set of specified bifurcation loci. In our study there is a surprising amount of complexity added by specifying the three fixedpoint bifurcation loci together, rather than one at a time.

We also provide a preliminary classification of the types of codimensionone bifurcations one should expect in general studies of families of twoparameter families of maps of the plane. Comments on numerical continuation techniques are provided as well.
\end{abstract}




\section{Introduction: Background and Motivation}

This paper is primarily a numerical study of certain properties of the family of maps of the complex plane

$$
f_{(C, A)}(z)=z^{2}+z+C+A \bar{z},
$$

where $z$ is a complex dynamic (phase) variable, $\bar{z}$ its complex conjugate, and $C$ and $A$ are complex parameters. In particular, we study the loci of the bifurcations of the fixed points present in this family: the saddle-node, period-doubling and Hopf bifurcations.

For $A=0$, the family is equivalent to the complex quadratic family, $Q_{\tilde{C}}$ : $z \mapsto z^{2}+\tilde{C}$, but for nonzero $A$, our family is non-complex-analytic. So it makes more sense to view the whole family as maps of $\mathcal{R}^{2}$ rather than as maps of $\mathcal{C}$. This is done by considering as real variables both the real and imaginary parts of $z$. Similarly, we view all complex parameters as two real parameters. Our family can then be considered as a four-real-parameter family of maps of the real plane. We prefer, however, for reasons discussed below, to consider it as a two-parameter family of two-parameter families of maps of the plane. In this context $C$ codes the two real primary parameters and $A$ codes the two real auxiliary parameters.

Our model family is of interest for a variety of reasons. Not the least is that it contains the family $f_{(C, 0)}$ which is equivalent to the quadratic family, $Q_{\tilde{C}}$. See subsection 1.1 for details on the equivalence. The family $f_{(C, 0)}$ demonstrates a fascinating richness on its own. This is evident in particular in the Mandelbrot set $\mathcal{M}$ in the $C$ plane, defined as the set of $C$ values for which the orbit of the critical point under $f_{(C, 0)}$ stays bounded. Many facts about the intricate structure of $\mathcal{M}$ are known. For example, the interior of $\mathcal{M}$ contains an infinity of bulbs (hyperbolic components), each bulb corresponding to maps with an attracting periodic orbit of a given period. That the union of the bulbs gives the whole interior of $\mathcal{M}$ is widely conjectured, but to our knowledge still unknown (Carleson and Gamelin [1993], for example). As such, a complete bifurcation study of our model family, which includes the complex quadratic family, is necessarily impractical. Some restrictions are necessary in order to design a more practical study.

One possibility is to restrict our auxiliary parameter $A$ to be small and/or real. Studies in this direction include Bielefeld, Sutherland, Tangerman and Veerman [1993] $\left(r^{2 \alpha} e^{2 i \theta}+\tilde{C}\right)$, Drexler [1996] $\left(z^{2}+\tilde{C}+\alpha x\right)$, and Peckham [1998] $\left(z^{2}+\tilde{C}+\alpha \bar{z}\right)$. In all three studies, the parameter $\alpha$ is real, and for $\alpha=1,0,0$, respectively, each corresponding family is the quadratic family $z^{2}+\tilde{C}$.

Our choice, however, was to restrict the types of bifurcations we considered. The natural first choice for us was to restrict to local fixed-point bifurcations: saddle-nodes, period doublings, and Hopf bifurcations. This drastic restriction still left us with an incredible number and complexity of phenomena to study. 
With regard to the Mandelbrot set, this restricts our attention to only its main cardioid. Inside this cardioid, each corresponding map has an attracting fixed point. On the cardioid itself, each corresponding map has a fixed point with neutral linear stability. As $A$ is perturbed from zero, the cardioid undergoes an interesting evolution, largely as the Hopf bifurcation curve of the perturbed families. The bifurcation evolution (as $A$ varies from zero) in a neighborhood of each $C$ value on the cardioid is fascinating, but by restricting to only fixedpoint phenomena, two points are distinguished from the rest: the cusp point at $C=0\left(\tilde{C}=\frac{1}{4}\right.$ in $\left.z^{2}+\tilde{C}\right)$, which we call the saddle-node point in this paper, and the point halfway around the cardioid at $C=-1\left(\tilde{C}=-\frac{3}{4}\right.$ in $\left.z^{2}+\tilde{C}\right)$, called the period-doubling point. These two points are distinguished because fixed-point bifurcations grow from them as $A$ is perturbed from zero: a fixedpoint saddle-node curve evolves from the saddle-node point and a fixed-point period-doubling curve evolves from the other (period-doubling) point. It is the interaction of these three bifurcation curves: saddle-node, period-doubling, and Hopf, that occupies most of this paper.

Interest in the saddle-node point in particular led to the form of our model family: $z+z^{2}+C+A \bar{z}$ is obtained from $z^{2}+C+A \bar{z}$ by translating the saddle-node point (in both $C$ and $z$ ) to the origin. The $A \bar{z}$ perturbing term was chosen as the simplest one which breaks the complex analyticity of the family. The complex parameter $A$ was deemed necessary because our early numerical studies showed that several bifurcation scenarios which were obtained for complex values of $A$ were not obtainable for real values of $A$. This was true even when we considered phenomena which were restricted to a neighborhood of the saddle-node point. That is, the numerics indicated the real codimension of the cusp point was at least two (in the auxiliary parameter space). This is also consistent with the fact that the linearization at the saddle-node fixed point, being the identity, is of codimension four (in the total parameter space - primary plus auxiliary). Thus a four-total-parameter unfolding, which we have via the complex parameters $C$ and $A$, was for us, necessary.

Separating our parameters as primary vs. auxiliary coincides with the treatment adopted by Golubitsky and Schaeffer [1985], where they identify a single primary bifurcation parameter and view all other parameters as auxiliary parameters. In our work it seems more natural to designate two primary parameters. This is largely because of the original motivation: studying maps which are non-complex-analytic perturbations of one-complex-parameter families, such as $z^{2}+\tilde{C}$. The original complex parameter $\left(\tilde{C}\right.$ in $\left.z^{2}+\tilde{C}\right)$ gives a natural choice for the two real primary parameters.

The general goal of the study of our family $f_{(C, A)}$ is to understand how bifurcation diagrams in the $C$ plane change as the auxiliary parameter $A$ is varied. More precisely, we would like to divide the $A$ plane into equivalence classes, where equivalence of two $A$ values $\hat{A}$ and $\tilde{A}$ is defined by having equivalent corresponding $C$ plane bifurcation diagrams. There are, however, many ways to 
define equivalence of $C$ plane bifurcation diagrams. Our notion of equivalence with respect to specified bifurcation phenomena is further described at the beginning of section 2. The loci we deal with are fixed-point saddle-nodes, fixed-point period-doublings, and fixed-point (extended) Hopf bifurcations. We first treat each of these sets individually, then together. There is a surprising amount of complexity added to the study when loci are grouped together.

What we believe to be new in this paper are the use of a complex rather than real auxiliary parameter, the idea of equivalence with respect to specified bifurcation loci, the numerical results for our family, including a global division of the auxiliary parameter space (we consider all $A$ values in the plane), the classification of types of bifurcations present in more general families of two-realparameter-families of maps of the plane, and the relationship between certain codimension-one phenomena in the space of two-real-parameter-families of maps of the plane to codimension-three phenomena in the space of maps of the plane. Some of the continuation techniques may also be new.

The rest of the paper is organized as follows. In the rest of the introduction, we relate our model to the quadratic family ( $\sec 1.1$ ), and then present a few of the results of a previous study [Peckham, 1998] to better explain the setting and the context of the current study (sec 1.2). In sec 2, we present our case study of the model family. We derive explicit formulas for our three bifurcation loci, and we use these formulas to determine certain bifurcation curves in the $A$ plane. Other $A$-plane bifurcation curves are located and computed numerically. In sec 3 we classify the bifurcations which are codimension-one in the auxiliary parameter space. In sec 4 we comment on the continuation algorithms used to compute the bifurcation diagrams in the paper.

\subsection{The complex quadratic family}

Note that when $A=0$ our family $f_{(C, A)}(z)=z^{2}+z+C+A \bar{z}$, is equivalent (conjugate) to what is usually referred to as the complex quadratic family $Q_{(\tilde{C}, 0)}(w)=w^{2}+\tilde{C}$ via a change of parameter: $f_{(C, 0)} \simeq Q_{(C+1 / 4,0)}$, where the equivalence is given by the translation $w=z+\frac{1}{2}$. The form $z+z^{2}+C$ is more suited to our purposes because it moves a primary point of interest, the "saddle-node point" of the complex quadratic family, to the origin in both variables $z$ and $C$.

Furthermore, when either quadratic family, $f_{(C, 0)}$ or $Q_{\tilde{C}, 0)}$, is perturbed with the simplest possible non-complex-analytic term, $A \bar{z}$ (resp. $A \bar{w}$ ), the resulting families $f_{(C, A)}(z)$ and $Q_{(\tilde{C}, A)}(w) \equiv w^{2}+\tilde{C}+A \bar{w}$, are conjugate, again by the translation $w=h(z)=z+\frac{1}{2}$. That is, $h \circ f_{(C, A)}=Q_{\left(C+\frac{1}{4}-\frac{A}{2}, A\right)} \circ h$. Thus, for any fixed $A$, the geometry of the $C$ plane bifurcation sets for the $f_{(C, A)}$ is a translation of the bifurcation sets for $Q_{(\tilde{C}, A)}$. 


\subsection{Points to regions}

We now recall a few of the results of previous work [Peckham, 1998] to better describe the setting and context of the current study. More complete explanations are included in that reference. Figure 1 summarizes the results of that work. Figures $1 \mathrm{a}$ and $1 \mathrm{~b}$ show partial $C$-plane bifurcation diagrams for the family $Q_{(C, A)}$ for $A=0$ and $A=-0.1$, respectively. By the above subsection, these bifurcation diagrams are translates of those for the family $f_{(C, A)}(z)$ studied in this paper.

Place Figure 1 near here.

The primary $C$-plane bifurcation diagram for $A=0$ are the boundaries of the bulbs (i.e., hyperbolic components) of the Mandlebrot set. When considered as a two-real-parameter family of maps of the plane, the bulb boundaries are Hopf bifurcation curves. Some of these bulb boundaries are shown in Fig. 1a. When $A \neq 0$, these bulb boundaries evolve into much more interesting bifurcation scenarios. More specifically, certain bifurcation points in the Mandlebrot set (the cusp $H^{0 / 1}$ of the main cardioid and the contact points between bulbs such as $H^{1 / 2}$ ) evolve into bifurcation regions. An example, with $A=-0.1$, is shown in Fig. 1b. All curves shown are local periodic-point bifurcations: saddle-nodes, period-doublings, or Hopfs. They are determined by the following equations, respectively, where $Q_{\tilde{C}, A}$ is the four-real-parameter family of maps of the plane defined above in sec 1.1 (interpreting the complex plane as equivalent to $\mathcal{R}^{2}$ ) and $D Q_{(\tilde{C}, A)}$ is its two-by-two Jacobian derivative matrix. Recall that $A$ is fixed in each part of Fig. 1.

$$
\begin{array}{cc}
Q_{(\tilde{C}, A)}^{n}(x, y)-(x, y) & =0 \\
1-\operatorname{tr}\left(D Q_{(\tilde{C}, A)}^{n}(x, y)\right)+\operatorname{det}\left(D Q_{(\tilde{C}, A)}^{n}(x, y)\right) & =0 \\
Q_{(\tilde{C}, A)}^{n}(x, y)-(x, y) & =0 \\
1+\operatorname{tr}\left(D Q_{(\tilde{C}, A)}^{n}(x, y)\right)+\operatorname{det}\left(D Q_{(\tilde{C}, A)}^{n}(x, y)\right) & =0 \\
Q_{(\tilde{C}, A)}^{n}(x, y)-(x, y)=0 \\
\operatorname{det}\left(D Q_{(\tilde{C}, A)}^{n}(x, y)\right)-1=0 \\
\left|\operatorname{tr}\left(D Q_{(\tilde{C}, A)}^{n}(x, y)\right)\right|<2 .
\end{array}
$$

The first vector equation for all three loci is equivalent to two scalar equations and requires $(x, y)$ to be a period- $n$ point of $Q_{(\tilde{C}, A)}$. The remaining equations are restrictions on the eigenvalues. They are obtained by recalling that an eigenvalue $\lambda$ of a $2 \times 2$ matrix $B$ satisfies $\lambda^{2}-\operatorname{tr}(B) \lambda+\operatorname{det}(B)=0$. Thus the period- $n$ saddle-node $\left(S N_{n}\right)$ requires an eigenvalue of one for $D Q_{(\tilde{C}, A)}$, the period- $n$ period-doubling $\left(P D_{n}\right)$ requires an eigenvalue of negative one for $D Q_{(\tilde{C}, A)}$, and the period- $n$ Hopf $\left(H_{n}\right)$ requires $D Q_{(\tilde{C}, A)}$ to have complex conjugate eigenvalues 
on the unit circle. Nondegeneracy conditions which would ensure nodegenerate bifurcations are not considered here. Note that the Hopf condition can be reformulated by requiring the product of the two eigenvalues to be one and the trace to be less than two in magnitude. Without the trace restriction, the product of the eigenvalues equalling one would extend the true Hopf set to also include nonbifurcation points which are saddles with eigenvalues such as 2 and 1/2. The label $\left(E H_{n}\right)$ stands for this period-n Extended Hopf locus. It is the algebraic closure of the Hopf locus.

As explained, for example in Peckham [1998], saddle-node and period-doubling loci are points in generic one-complex-parameter families of complex analytic maps of the complex plane, but curves in generic two-real-parameter families of analytic (or $C^{\infty}$ or $C^{k}$ ) maps of the real plane. One of the primary differences between Fig. 1a and Fig. 1b is the presence in the latter figure of saddle-node and period-doubling curves. For example, there is a circle of fixed-point perioddoubling points $\left(P D_{1}\right)$ in Fig. 1b which has grown out of the period-doubling point (the contact point $H^{1 / 2}$ between the main cardioid and the period-two bulb in Fig. 1a). Similarly, there is a triangular curve of saddle-node points $\left(S N_{1}\right)$ in Fig. 1b near where the cusp, $H^{0 / 1}$ of Fig. 1a's main cardioid was. (The triangular saddle-node curve is barely visible, but if it were enlarged, it would be topologically equivalent to the triangular saddle-node curve and nearby extended Hopf curve displayed in $C$-plane 1 in Fig. 9b.) All other period- $n$ bulbs in Fig. 1b have been deformed from Fig. 1a, and the bulb contact points of 1a have evolved into period- $n$ saddle-node curves, $S N_{n}$. Details are in Peckham [1998].

In one sense this paper narrows the focus of Peckham [1998] by restricting attention to only the fixed-point bifurcations. That is, of the bifurcations in Fig. 1a, this paper deals only with the fixed-point (extended) Hopf curve $E H_{1}$, the saddle-node point $H^{0 / 1}$, and the period-doubling point $H^{1 / 2}$; of the bifurcations in Fig. 1b, this paper deals only with the fixed-point extended Hopf curve $E H_{1}$, the fixed-point period-doubling curve $P D_{1}$, and the fixed-point saddle-node curve $S N_{1}$. On the other hand, this restriction to local fixed-point bifurcations allows us to more easily broaden the consideration of the auxiliary parameter: we consider all values of the complex auxiliary parameter $A$ rather than merely values which are real and close to $A=0$.

\section{Equivalence w.r.t. specified bifurcation loci}

In this section, we study the model family of maps $f_{(C, A)}: \mathcal{R}^{2} \rightarrow \mathcal{R}^{2}$, recopied from the Introduction:

$$
f_{(C, A)}(z)=z^{2}+z+C+A \bar{z} .
$$

We describe the evolution of the three principal local fixed-point $C$-plane bifurcation loci: saddle-nodes, period-doublings, and (extended) Hopfs as the 
auxiliary parameter $A$ is varied. Of course restricting to these three bifurcations ignores many bifurcation aspects of the problem, but as we shall see, the information contained in these sets includes much more than one might at first suspect.

Note that any one of our bifurcation points is really associated to a single point in the six-real-dimensional space coded by $(z, C, A)$. It is the ways in which, for fixed values of the auxiliary parameter $A$, these bifurcation points project to the $C$ parameter plane that we wish to now classify. We will view two $A$ values as equivalent with respect to a specific collection of bifurcation loci if the collection of bifurcation loci projected to the $C$ plane "looks the same." The following definition makes "looks the same" more precise, although we use it only as a guide for our bifurcation study. We don't actually prove that bifurcation diagrams which we view as equivalent are, in fact, equivalent.

Definition 2.1 Let $g_{(C, A)}(x)$ be an $k+l$ parameter family of maps of $\mathcal{R}^{n}$ where $C \in \mathcal{R}^{k}, A \in \mathcal{R}^{l}$, and $x \in \mathcal{R}^{n}$. Assume $C$ codes the primary parameters and $A$ codes the auxiliary parameters. Two auxiliary parameters $\hat{A}$ and $\tilde{A}$ are equivalent with respect to a specific collection of bifurcation loci if there is a diffeomorphism $h: \mathcal{R}^{k} \rightarrow \mathcal{R}^{k}$ which maps the $C$ space for $\hat{A}$ to the $C$ space for $\tilde{A}$ and maps the corresponding bifurcation loci in the $C$ plane for $\hat{A}$ to their respective counterparts in the $C$ plane for $\tilde{A}$.

We remark that our definition of equivalence is of course only one of many possible notions one might employ. At one end of the spectrum would be the establishment of a diffeomorphism $h$ for which $f_{(C, \hat{A})}$ and $f_{(h(C), \tilde{A})}$ are topologically conjugate. That is, $f_{(C, \hat{A})}(z)=\phi_{C}^{-1} \circ f_{(h(C), \tilde{A})} \circ \phi_{C}(z)$, with $\phi_{C}$ a two-real-parameter family of homeomorphisms of $\mathcal{R}^{2}$ which varies at least continuously with $C$. Of course, establishing topological conjugacy for even one pair of maps of the plane is in general a difficult problem, so doing this for pairs of whole two-parameter families of maps, except in special cases, is not very practical.

Note that if two families $f_{(C, \hat{A})}$ and $f_{(h(C), \tilde{A})}$ were shown to be topologically conjugate, then the diffeomorphism $h$ would necessarily map bifurcation sets in the $C$ plane for $\hat{A}$ to corresponding bifurcation sets in the $C$ plane for $\tilde{A}$. For example, the set of fixed-point saddle-node bifurcations in the $C$ plane for $\hat{A}$ would map to the fixed-point saddle-node bifurcations in the $C$ plane for $\tilde{A}$. One way to weaken the equivalence defined by topological conjugacy would be to merely require that $h$ map all corresponding bifurcation sets to each other, but not require that the corresponding maps, $f_{(C, \hat{A})}$ and $f_{(h(C), \tilde{A})}$, be topologically conjugate.

Since a typical family has an infinity of bifurcation curves: saddle-nodes, period-doublings and Hopf bifurcations for all periods, not to mention a multitude of global bifurcations, requiring $h$ to map all corresponding bifurcation sets to each other is still an impractical requirement. Instead, we restrict attention 
to a specified set of bifurcation loci. Note that the larger the set of bifurcation loci specified, the finer the division of the $A$ plane into equivalence classes.

Our definition requires $h$ to be a diffeomorphism rather than a homeomorphism because we want cusps in the primary parameter plane (the $C$ plane) to be preserved. The smoothness of $h$ should in general match the smoothness of the maps under consideration. Orientation preservation for $h$ may desirable in some cases, but we do not require it in our definition. The idea of the definition is motivated by wanting $g_{(C, \hat{A})}$ and $g_{(h(C), \tilde{A})}$ to have the same number and stability of period- $n$ points when the specified collection of bifurcation loci includes all three local period- $n$ bifurcations: saddle-nodes, period-doublings and Hopfs. This correspondence is not guaranteed in general by the definition, but is satisfied when $\hat{A}$ and $\tilde{A}$ are in the same connected component of equivalence classes of the auxiliary parameter space.

In our model family, the dimensions of the phase space $(n)$, the primary parameter space $(k)$, and the auxiliary space $(l)$ are all two. Note that with this heierarchical approach for four real parameters coded by the two complex parameters $C$ and $A$, curves such as the saddle-node, period-doubling and Hopf bifurcations in the $C$ plane will describe bifurcations which are codimension-one in the traditional sense (with no distinguished parameters), while curves in the $A$ plane will describe codimension-one phenomena in the space of two-parameter families of maps (that is, codimension-one in the auxiliary parameter space). These codimension-one phenomena in two-parameter families, when they correspond to traditional bifurcations, are typically codimension-three phenomena in the traditional sense.

Because the quadratic family admits explicit calculation, we provide many explicit formulas for bifurcation sets in the this section. In particular, we provide both the equations defining our bifurcation sets and explicit parametric representations of their solutions.

We first treat each bifurcation locus separately; then we treat them as a group. Specifically, we discuss $A$-plane bifurcation diagrams for projections of the following loci to the $C$-plane: Saddle-node (sec. 2.1), Period-doubling (sec 2.2), Extended Hopf (sec 2.3), Hopf (sec 2.4), Saddle-node-Period-doublingExtended Hopf (sec 2.5), and Saddle-node-Period-doubling-Hopf (sec 2.6). In sec 2.7, we "zoom in" on a special point in the Saddle-node-Period-doublingHopf $A$ plane: the local "saddle-node" bifurcation.

Preliminary calculations. Since all of our bifurcations involve fixed points, we first describe that locus. The map $f_{(C, A)}(z)=z+z^{2}+C+A \bar{z}$ has a fixed point $z$ if

$$
\tilde{f}_{(C, A)}(z):=z^{2}+C+A \bar{z}=0,
$$

The fixed points in $\mathcal{R}^{2} \times \mathcal{R}^{2} \times \mathcal{R}^{2}$ can thus be parametrized by $(A, z) \in \mathcal{R}^{2} \times \mathcal{R}^{2}$ :

$$
(z, A) \mapsto(z, C, A)=\left(z,-z^{2}-A \bar{z}, A\right), \quad\left(F P_{p a r}\right)
$$


and so defines a 4-dimensional submanifold of $\mathcal{R}^{2} \times \mathcal{R}^{2} \times \mathcal{R}^{2}$.

For each value of $A$, the fixed point set is a 2-dimensional manifold (surface), parametrized by $z$. For $A=0$ the projection of this surface from $(z, C)$ space to the $C$ plane is two-to-one, except at $C=0$ where it is one-to-one. For any fixed nonzero $A$, the projection of the surface to the $C$-plane is two- three- or four-to-one. We will see in sec 2.1 that for nonzero $A$ there is a triangular deltoid curve of saddle-nodes in the $C$ plane. The projection of the fixed-point surface is four-to-one for $C$ values inside the deltoid, two-to-one outside the deltoid, two-to-one at the three cusp points of the deltoid, and three-to-one on the rest of the deltoid. This allows many intersections of loci to be introduced when the loci are projected from the four-real-dimensional $(z, C)$ space to the two-real-dimensional $C$ plane, leading to numerous distinct $A$-plane equivalence classes.

To describe the three bifurcation sets, it is useful to have the expression for the differential of $f_{(C, A)}$ :

$$
D f_{(C, A)}=\left[\begin{array}{cc}
2 x+1+A_{1} & -2 y+A_{2} \\
2 y+A_{2} & 2 x+1-A_{1}
\end{array}\right],
$$

where $z=x+i y, A=A_{1}+i A_{2}$ and $C=C_{1}+i C_{2}$. This is most easily obtained by writing our map $f_{(C, A)}$ in rectangular coordinates:

$$
(x, y) \mapsto\left(x+x^{2}-y^{2}+C_{1}+A_{1} x+A_{2} y, y+2 x y+C_{2}+A_{2} x-A_{1} y\right)
$$

and computing the Jacobian derivative matrix. We will also use $z=r_{z} e^{i \theta_{z}}$ and $A=a e^{i \theta_{A}}$.

\subsection{Saddle-node locus.}

A fixed point of $f_{(C, A)}$ with an eigenvalue of one is generally referred to as a saddle-node point, and corresponds to a degenerate zero of $\tilde{f}_{(C, A)}$. Such points satisfy

$$
\left\{\begin{array}{cc}
z^{2}+C+A \bar{z} & =0 \\
4|z|^{2}-|A|^{2} & =0
\end{array}\right.
$$

The first equation ensures that $z$ is a fixed point of $f_{(C, A)}$. The second ensures an eigenvalue of one; it is derived from setting $1-\operatorname{tr}\left(D f_{(C, A)}\right)+\operatorname{det}\left(D f_{(C, A)}\right)=0$. Compare $(S N)$ to $\left(S N_{1}\right)$ in sec 1.2. We ignore any conditions which would ensure the nondegeneracy of the saddle-node bifucation.

For $A=0$, the only saddle-node point is at $(z, C)=(0,0)$. If $A \neq 0$, the second equation describes a circle in the $z$ plane with center at the origin and radius $|A| / 2$. See the projection to the $z$ plane in Fig. 2. It can be parametrized by $\theta_{z} \in[0,2 \pi) \simeq \mathcal{S}$. In the full phase $\times$ parameter space the saddle-node set is parametrized by $\left(A, \theta_{z}\right) \in \mathcal{R}^{2} \times \mathcal{S}$, identifying all angles $\theta_{z}$ at $A=0$ :

$$
\left(A, \theta_{z}\right) \mapsto(z, C, A)=\left(\frac{|A|}{2} e^{i \theta_{z}},-\frac{|A|^{2}}{4} e^{2 i \theta_{z}}-A \frac{|A|}{2} e^{-i \theta_{z}}, A\right) . \quad\left(S N_{p a r}\right)
$$


Place Figure 2 near here.

Place Figure 3 near here.

The saddle-node curve projected to the $C$ plane is equivalent for all nonzero values of $A$. It is a hypocycloid of three cusps. Its size depends on $|A|$ and its rotational orientation depends on $\arg (A)$. The only distinguished $A$ value, as illustrated in Fig. 3a, is thus the origin. For all nonzero values of $A$, we can find a diffeomorphism $h$ which maps the saddle-node set for $f_{(C, 1)}$ (the arbitrarily chosen equivalence class representative) to the saddle-node set for $f_{(C, A)}$. In fact, the diffeomorphism can be explicitly expressed as

$$
h(C)=a^{2} C e^{\frac{2 i \theta_{A}}{3}},
$$

where $A$ in polar coordinates is $a e^{i \theta_{A}}$ These statements can be verified by considering the parametric expression $S N_{\text {par }}$ above. It indicates that, for any fixed value of $A$, the projection of the saddle-node set to the $C$ parameter plane is

$$
C=-\frac{|A|^{2}}{4} e^{2 i \theta_{z}}-A \frac{|A|}{2} e^{-i \theta_{z}}
$$

$\theta_{z} \in[0,2 \pi)$, which is a classical parametrization of a deltoid curve (a hypocycloid of 3 cusps). Note that the full saddle-node set as determined by (SN) is invariant under the two substitutions

$$
(z, C, A) \mapsto\left(z e^{\frac{i \theta}{3}}, C e^{\frac{2 i \theta}{3}}, A e^{i \theta}\right), \quad \theta \in \mathcal{S},
$$

and

$$
(z, C, A) \mapsto\left(a z e^{i \theta}, a^{2} C e^{2 i \theta}, a A e^{3 i \theta}\right), \quad a \in[0, \infty)
$$

The first invariance implies that as the origin in the $A$ plane is circled once, the saddle-node deltoid makes $2 / 3$ of a complete rotation in the $C$ plane. This is illustrated in Fig. 3b, $C$ planes 2 and 3. The corresponding $C$ plane figure has rotated by $1 / 12$ of a full rotation while the argument of $A$ has changed by $1 / 8$ of a full rotation. Together, the two invariant substitutions imply the formula given above for $h$.

Saddle-node Cusps. The two invariances above, along with the observation that for $A$ real and positive $(A=|A|)$ a cusp occurs at $\theta_{z}=0$, lead to the set of saddle-node cusps being a topological disk, parametrized by $\left(a, \theta_{z}\right) \in[0, \infty) \times \mathcal{S}$, identifying all angles $\theta_{z}$ at $a=0$ :

$$
\left(a, \theta_{z}\right) \mapsto\left(\frac{a e^{i \theta_{z}}}{2}, \frac{-3 a^{2} e^{2 i \theta_{z}}}{4}, a e^{3 i \theta_{z}}\right) \quad(\text { SNcusp par })
$$


Note that there are three cusps in the $C$ plane for each fixed nonzero $A$. Since the cusps persist as the auxiliary parameter $A$ is varied, they are classified as codimension-zero in the auxiliary parameter space. This is consistent with the traditional no-distinguished-parameter classification of a cusp as a codimensiontwo phenomenon: an eigenvalue one and a higher order degeneracy. We will see later that, in contrast, cusps on the period-doubling and Extended Hopf curves are codimension-one in the auxiliary parameter space.

\subsection{Period-doubling locus.}

A fixed point of a map $f: \mathcal{R}^{2} \rightarrow \mathcal{R}^{2}$ is a period doubling point if one of the eigenvalues of $D f_{(C, A)}$ is equal to -1 . Thus we define the period doubling locus to be,

$$
\left\{\begin{array}{ccc}
z^{2}+C+A \bar{z} & = & 0 \\
4|z+1|^{2}-|A|^{2} & = & 0
\end{array}\right.
$$

Compare $(P D)$ to $\left(P D_{1}\right)$ in sec 1.2. The second equation, derived from $1+$ $\operatorname{tr}\left(D f_{(C, A)}\right)+\operatorname{det}\left(D f_{(C, A)}\right)=0$, ensures an eigenvalue of negative one. Nondegeneracy conditions are not considered. The second equation implies that for each fixed value of $A$, the period-doubling points project to a circle in the $z$ plane with center -1 and radius $|A| / 2$, as illustrated in Fig. 2. Labelling the angle of this circle $\theta_{D} \in[0,2 \pi) \simeq \mathcal{S}$, we can parametrically represent these points for $\left(A, \theta_{D}\right) \in \mathcal{R}^{2} \times \mathcal{S}$, identifying all angles $\theta_{D}$ at $A=0$, as:

$$
\left(A, \theta_{D}\right) \mapsto(z, C, A)=\left(-1+\frac{|A|}{2} e^{i \theta_{D}},-\left(-1+\frac{|A|}{2} e^{i \theta_{D}}\right)^{2}-A\left(-1+\frac{|A|}{2} e^{-i \theta_{D}}\right), A\right) .
$$

Place Figure 4 near here.

The parametrization implies that for each fixed nonzero value of $A$, the period-doubling set in the four-real-dimensional $(z, C)$ space is a topological circle. This topological circle always projects to a geometric circle in the $z$ plane. The projection to the $C$ plane, however, varies greatly with $A$. It projects as a topological circle for small values of $A$, but for larger values of $A$ the projection can change its topology at points where the curve either develops a cusp, or a self tangency. The $A$-plane bifurcation diagram and representative $C$ planes are shown in Fig. 4. There is one curve in the $A$ plane corresponding to self tangent projections of the $\mathrm{PD}$ curve $(\mathrm{C}$ planes 6,10$)$, and a line in the $A$ plane corresponding to PD curves with cusps (C planes, 2,8,9). The two endpoints of the self tangent projection curve, where the curve terminates on the PD cusp line, are "rhomboid" cusp points - cusp points with an additional higher order degeneracy $(\mathrm{C}$ plane 8$)$. The origin is distinguished because the period-doubling locus has shrunk to a point (C plane 4). This leaves us with three generic regions, 
distinguished by the number of self intersections of the period-doubling locus: 0 (C planes 3,5), 1 (C plane 1), 2 (C plane 7).

Remarks. The line of period-doubling cusps in the $A$ plane indicates that this set is codimension-one in the auxiliary parameter space, so it is quite different from the saddle-node cusps, which were codimension-zero in the auxiliary parameter space. It turns out that the period-doubling cusps correspond to a traditional codimension-three local bifurcation: a one-negative-one eigenvalue point (ONO) with a specific degeneracy in the quadratic terms of its normal form. Because of this, we can compute it's formula directly. We do so below in sec 3 .

The curve of period-doubling self tangencies was located and computed numerically. It has no corresponding traditional bifurcation since it involves two period-doubling bifurcations which are located at two distinct $z$ values; they just happen to also have the same $C$ value, so when projected to the $C$ plane, they "interact."

\subsection{Extended Hopf locus.}

A fixed point of a map $f: \mathcal{R}^{2} \rightarrow \mathcal{R}^{2}$ is a Hopf point if the eigenvalues lie on the unit circle, that is, if $\operatorname{det}\left(D f_{(C, A)}\right)=1$ and $\left|\operatorname{tr} D f_{(C, A)}\right|<2$. These equations become,

$$
\left\{\begin{aligned}
z^{2}+C+A \bar{z} & =0 \\
4|z+1 / 2|^{2} & =|A|^{2}+1
\end{aligned}\right\}(E H)
$$

As in the Introduction, we call the locus without the inequality the Extended Hopf locus. Compare $(H)$ and $(E H)$ here to $\left(H_{1}\right)$ and $\left(E H_{1}\right)$ in sec 1.2. For fixed $A$, the Extended Hopf set also projects to a circle in the phase plane. It has center $-\frac{1}{2}$ and radius $\frac{\sqrt{|A|^{2}+1}}{2}$, as illustrated in Fig. 2 . It is most naturally parametrized by the angle in this circle, which we label $\theta_{H}$. Its parametric representation, for $\left(A, \theta_{H}\right) \in \mathcal{R}^{2} \times \mathcal{S}$, is $\left(A, \theta_{H}\right) \mapsto(z, C, A)$ with

$$
\left\{\begin{aligned}
& z=\left(-\frac{1}{2}+\frac{\sqrt{|A|^{2}+1}}{2} e^{i \theta_{H}},\right. \\
& C=-\left(-\frac{1}{2}+\frac{\sqrt{|A|^{2}+1}}{2} e^{i \theta_{H}}\right)^{2}-A\left(-\frac{1}{2}+\frac{\sqrt{|A|^{2}+1}}{2} e^{-i \theta_{H}}\right) . \\
&\left(E H_{\text {par }}, H_{\text {par }}\right)
\end{aligned}\right.
$$

The parametric formulas are identical for both the Extended Hopf and true Hopf sets, but the parameter $\theta_{H}$ is restricted to two arcs on the circle for the true Hopf set: $\theta_{H} \in\left(\theta_{T B}, \pi-\theta_{T B}\right) \cup\left(\pi+\theta_{T B}, 2 \pi-\theta_{T B}\right)$, where $\theta_{T B}=\arctan (|A|)$. More details are in sec 2.4.

Place Figure 5 near here. 
The bifurcation diagram and corresponding $C$ planes are shown in Fig. 5 . There is one curve each in the $A$ plane corresponding to self tangent projections of the EH curve (C planes 6,10,15), cusps on the EH curve (C planes $2,8,12,13,14)$, and triple intersection of EH points (C plane 4). Note that the $A$ plane's EH cusp curve goes through the origin. This is consistent with the fact that that at $A=0$ the $\mathrm{EH}$ curve is the main cardioid of the Mandlebrot set (C plane 8), which does include a cusp. There are three labelled codimension-two points: at $A$ values labelled by numbers 2,6 , and 12 . At number 2 , the corresponding $\mathrm{EH}$ curve in the $\mathrm{C}$ plane has two distinct cusps; at number 6, the two points of the EH curve that are self tangent also have the same curvature; at number 12, the EH curve has a cusp that also projects to another point on the $\mathrm{EH}$ curve. The bifurcation curves divide the A plane into six generic regions, partially distinguished by the number of self intersections: 0 (C plane 9$), 1$ (C planes 1,7), 2 (C plane 11), and 3 (C planes 3,4 ). $\mathrm{C}$ planes 1 and 7 can be distinguished by the winding number as one travels around the $\mathrm{EH}$ locus, but the winding number does not distinguish between $\mathrm{C}$ planes 3 and 5 .

Remarks. Similar to the period-doubling cusp curve, the $A$ plane's EH cusp curve corresponds (except at $A=0$ ) to a traditional codimension-three bifurcation: a Takens-Bogdanov point with a specific degeneracy in the quadratic terms of its normal form. More details about this degeneracy are discussed below in sec 3 . The other two codimension-one bifurcation curves, the self tangent projections and the triple projection intersections, do not correspond to traditional codimension-three bifurcations. The former involves two $E H$ points with distinct $z$ values; the latter involves three $E H$ points with distinct $z$ values.

\subsection{Hopf locus.}

The equations for Hopf points were already given by $(H)$ in the previous subsection. The equivalence classes for true Hopf bifurcations are, of course, related to the equivalence classes for the Extended Hopf bifurcations, since Hopf points are the Extended Hopf points that also satisfy the inequality in $(H)$ above. In order to identify which Extended Hopf points are true Hopf points, we must first investigate intersections of our three fixed-point bifurcation loci.

Pairwise intersections of bifurcation loci. The intersections of the three bifurcation loci is most easily seen in their projections to the phase plane. All three loci are circles and are pictured in Fig. 2 for $|A| \approx 0.5$. The only feature of Fig. 2 which depends on the argument of $A$ is the placement of the three points on the saddle-node curve which are cusp points when projected to the $C$-plane.

Since the fixed points are parametrized by $z$ and $A$ (recall eqn. $\left(F P_{p a r}\right)$ before sec 2.1), for any fixed $A$ value intersections in the projection to the $z$ plane will in fact be true intersections of $(z, C, A) \in \mathcal{R}^{6}$. It is obvious from the 
figures that intersections of the saddle-node and period-doubling curves can only occur if $|A| \geq 1$. The $x$ value at any intersection is $-\frac{1}{2}$. A short calculation shows that an intersection of the saddle-node set and the Hopf set requires $x=0$, and the intersection of the period-doubling set with the Hopf set requires $x=-1$. Using $\delta= \pm 1$ leads to the following parametrizations of simultaneous solutions.

The intersections of the saddle-node locus with the Extended Hopf locus are parametrically represented for $(A, \delta) \in \mathcal{R}^{2} \times \mathcal{Z}_{2}$, with $(0,1)$ and $(0,-1)$ identified, as:

$$
(A, \delta) \mapsto(z, C, A)=\left(\delta \frac{|A| i}{2}, \frac{|A|^{2}}{4}+\delta \frac{A|A| i}{2}, A\right) .
$$

These points must have both eigenvalues equal to one, and are traditionally called Takens-Bogdanov points (TB).

The intersections of the period-doubling locus with the Extended Hopf locus are parametrically represented for $(A, \delta) \in \mathcal{R}^{2} \times \mathcal{Z}_{2}$, with $(0,1)$ and $(0,-1)$ identified, as:

$$
\begin{gathered}
(A, \delta) \mapsto(z, C, A)= \\
\left(-1+\delta \frac{|A| i}{2},-\left(-1+\delta \frac{|A| i}{2}\right)^{2}-A\left(-1-\delta \frac{|A| i}{2}\right), A\right) .
\end{gathered}
$$

These points must have both eigenvalues equal to negative one, and are called double negative one points (DNO).

The intersections of the saddle-node locus with the period-doubling locus are parametrically represented for $(A, \delta) \in\left(\mathcal{R}^{2} \backslash\left\{A_{1}^{2}+A_{2}^{2}<1\right\}\right) \times \mathcal{Z}_{2}$, with $(A, 1)$ and $(A,-1)$ identified whenever $|A|=1$, as:

$$
\begin{gathered}
(A, \delta) \mapsto(z, C, A)= \\
\left(-\frac{1}{2}+\delta \frac{\sqrt{|A|^{2}-1} i}{2},-\left(-\frac{1}{2}+\delta \frac{\sqrt{|A|^{2}-1} i}{2}\right)^{2}-A\left(-\frac{1}{2}-\delta \frac{\sqrt{|A|^{2}-1} i}{2}\right), A\right),
\end{gathered}
$$

These points must have one eigenvalue of one and the other of negative one, and are called one-negative-one points (ONO).

Hopf vs. Extended Hopf points. It is now convenient to separate the Extended Hopf set into true Hopf bifurcation points (defined by (H) above) and its complement in the Extended Hopf set, the saddle points with real eigenvalues whose product is equal to one. The inequality in $(\mathrm{H})$, is easy to visualize in Figure 2. Thus the Hopf points are the Extended Hopf points which lie in the strip $-1<x<0$ in the $z$ plane. The two Takens-Bogdanov points, $T B_{1}$ and $T B_{2}$, and the two double negative one points, $D N O_{1}$ and $D N O_{2}$, mark the divisions between the Extended Hopf points which are true Hopfs points and those that are not true Hopf points. If $|A|$ were greater than one, the perioddoubling and saddle-node curves would intersect at two points we would label $\mathrm{ONO}_{1}$ and $\mathrm{ONO}_{2}$. 
As one traverses the $\mathrm{EH}$ circle counterclockwise from the $T B_{1}$, the eigenvalues of the corresponding fixed point for the original map start both at +1 . As the circle is followed across the strip $-1<x<0$, the eigenvalues separate, both remaining on the unit circle and each being the complex conjugate of the other. The eigenvalues arrive together at negative one as $z$ arrives at $D N O_{1}$. Continuing toward $\mathrm{DNO}_{2}$, the eigenvalues separate, but now are real and negative (and of course still satisfying the property that their eigenvalues multiply to one). They obtain a maximum separation at some point, then reverse direction back toward each other, and meet again at negative one as $z$ arrives at $\mathrm{DNO}_{2}$. The other half of the trip around the Extended Hopf circle is similar. The eigenvalues once again separate and run around opposite sides of the unit circle and meet at one when $z$ arrives at $T B_{2}$. They then separate as real positive eigenvalues, reach a maximum separation, head back toward each other, and meet again at one, as $z$ returns to its starting point at $T B_{1}$.

The parametric representation of the true Hopf points is the same as for the Extended Hopf points, except that the argument $\theta_{H}$ is restricted to trace out only the part of the circle that projects to the strip $-1<x<0: \theta_{H} \in$ $\left(\theta_{T B}, \pi-\theta_{T B}\right) \cup\left(\pi+\theta_{T B}, 2 \pi-\theta_{T B}\right)$, where $\theta_{T B}=\arctan (|A|)$.

Back to the Hopf locus. We now know that the true Hopf locus for any fixed (nonzero) A value is a pair of curve segments, each segment having a TakensBogdanov point at one end and a double-negative-one point at the other. The A-plane bifurcation diagram and corresponding Hopf curves in the $\mathrm{C}$ plane are shown in Fig. 6. In going from Fig. 5 to Fig. 6, we have eliminated parts of the Extended Hopf $A$-plane bifurcation curves that involve saddle points (rather than true Hopf points). None of the EH-EH-EH triple intersection curve survived; none of the EH cusp curve survived; only a small portion of the $\mathrm{EH}-\mathrm{EH}$ tangency curve survived to become the $\mathrm{H}-\mathrm{H}$ tangency curve: from $\mathrm{A}$ plane location 11 to 7 and its complex conjugate in the upper half plane. But we added curves where the endpoints of the Hopf curves project to the same $C$ value as some other Hopf point (TB-H-projection-intersections (C planes $5,9,11,12$ ) and DNO-H-projection-intersections (C planes 2,12)). Most of the phase portraits corresponding to the numbered points on $A$-plane bifurcation curves actually correspond to $A$ values which are codimension-two points in the auxiliary parameter space: $2-\mathrm{C}$ plane intersection of a a DNO point with another DNO point (instead of just a DNO point with a Hopf point), $5-\mathrm{C}$ plane intersections of two separate TB points with Hopf points, $7-\mathrm{C}$ plane self tangent intersection with curvature matching as well (same as $\mathrm{C}$ plane 6 in Fig. 5), 11 - C plane intersection of a TB point with a Hopf point with the further degeneracy that it is an endpoint of a curve of $\mathrm{C}$ plane self tangencies of Hopf points, and $12-\mathrm{C}$ plane intersection of a TB point and a DNO point.

The division of the A plane leaves us with six generic regions. We list them according to how many intersections there are of the two Hopf segments: 0 
(C planes 1,10), 1 (C planes 3,8), 2 (C plane 4 and its conjugate), 3 (C plane 6). $\mathrm{C}$ planes 1 and 10 are equivalent; $\mathrm{C}$ planes 3 and 8 are distinguished by the orientation of the crossing, assuming that the DNO endpoints of the Hopf segments are mapped to DNO endpoints and TB endpoints are mapped to TB endpoints.

Place Figure 6 near here.

\subsection{Saddle-node, Period-doubling, and Extended Hopf loci together (SN-PD-EH).}

We now view equivalence classes of $C$-plane bifurcation diagrams for diagrams which include all three bifurcation loci at once. Not surprisingly, the $A$-plane divisions are much finer than when each locus was considered individually. The result, along with only a few of the representative $C$-plane bifurcation diagrams, is shown in Fig. 7. There are thirty-three distinct regions, numbered in Fig. 7a, along the real $\mathrm{A}$ axis. Not numbered on the diagram are 300 regions we counted in the upper half $A$ plane and 300 "conjugate" regions in the lower half plane. This gives 633 distinct $A$-plane regions. This doesn't count points on the bifurcation curves. To get a feel for the information contained in the bifurcation diagram, an enlargement of path points 15 through 24 is shown in $7 \mathrm{~b}$, with corresponding $\mathrm{C}$ planes in Fig. 7c.

Between A values numbered 15 and 16, the left-hand saddle-node cusp pushes through the right-hand part of the period-doubling (curve s, SNcusp$\mathrm{PD}$ projection intersections). Between 16 and 17, the part of the $\mathrm{EH}$ curve between the two Takens-Bogdanov points pushes through the right-hand part of the period-doubling curve (curve o, EH-PD tangent projection intersections). Between 17 and 18, the left-hand saddle-node cusp pushes through the left-hand part of the Extended Hopf curve (curve j, SNcusp-EH projection intersections). Between 18 and 19, the two Takens-Bogdanov points cross from outside the period-doubling curve to inside it (curves $v$ and $\bar{v}$, TB-PD projection intersections). Between 19 and 20, three curves are crossed simultaneously on the real $A$ axis at $A=1$. The left hand saddle-node cusp pushes through the left-hand part of the period-doubling curve (a different part of the same SNcusp-PD curve $\mathrm{S}$ which is between A values 15 and 16); two parts of the Extended Hopf curve become tangent and push through (curve d, EH-EH tangent projections); and a pair of one-negative-one points is born (curve $t$, double ONO points). By Fig. 20 the two ONO points have separated from the real $\mathrm{C}$ axis where they were born at $\mathrm{A}=1$ and have travelled - one up and one down - along the right-hand side of the saddle-node deltoid toward the top and bottom cusps respectively of the deltoid). Between 20 and 21, the left-hand parts of the Extended Hopf and period-doubling curves have become tangent (curve l, PD-EH tangent projections). The bifurcation between 21 and 22 is difficult to see: the 
two one-negative-one points are to the right of the right-hand part of the Extended Hopf curve in $\mathrm{C}$ plane 21, but to the left of the right-hand part of the Extended Hopf curve in C plane 22. In between are curves $w$ and $\bar{w}$, ONO-EH projection intersections. Between 22 and 23, the right-hand part of the perioddoubling curve has pushed through the right-hand part of the Extended Hopf curve, crossing curve 0, the PD-EH tangent projection. Between 23 and 24, we again cross three curves simultaneously at $A=2$. Two of them are part of the same SNcusp-PD intersection curve labelled q; this bifurcation is difficult to see at the scale of Fig. 7c, but the two one-negative-one points are on the right-hand edge of the saddle-node deltoid in $\mathrm{C}$ plane 23 , but on the top and bottom edges, respectively, in $\mathrm{C}$ plane 24 . The third bifurcation, corresponding to $A$ values for which two parts of the $C$-plane period-doubling curve are tangent, is easily understood by comparing the period-doubling curves in C planes 23 and 24 .

We realize that more regions would need to be enlarged and a complete set of $C$-plane bifurcation diagrams provided to fully explain the bifurcations. But a full explanation is not our goal. We have merely tried to illustrate the kinds of bifurcations and complexity of bifurcation diagrams one would expect to encounter in such a study. Those interested in more details are encouraged to contact the authors.

Place Figure 7 near here.

\subsection{Saddle-node, Period-doubling, and Hopf loci together (SN-PD-H).}

This combination of three bifurcation loci is possibly the one of most relevance for understanding the dynamics of our model family. This is because, for this specified collection of bifurcation loci, families which have equivalent auxiliary parameters $A$, have the same qualitative dependence on the primary parameter $C$ with respect to the fixed points. That is, if $\hat{A}$ and $\tilde{A}$ are in the same connected equivalence class component with respect to fixed-point saddle-nodes, perioddoublings and Hopfs, then there is a diffeomorphism $h$ with maps the $C$-plane bifurcation loci for $A=\hat{A}$ to the bifurcation loci for $A=\tilde{A}$, and each pair of individual maps $f_{(C, \hat{A})}$ and $f_{(h(C), \tilde{A})}$ has the same number and corresponding stability of the respective fixed points.

Place Figure 8 near here.

It turns out that the SN-PD-H bifurcation diagram is obtained from the SN-PD-EH bifurcation diagram by removing portions of $A$-plane bifurcation curves for which the corresponding $C$-plane bifurcation curves involve $\mathrm{EH}$ points which are saddles rather than true Hopf points. In all cases, the $A$-plane bifurcations with " $\mathrm{H}$ " are contained in the corresponding $A$-plane bifurcations 
with "EH." More specifically, we remove all of curves $e$ and $l$, and portions of curves $d, h, j, m, \bar{m}, n, o, w$, and $\bar{w}$. No new curves are added in going from the SN-PD-EH diagram to the SN-PD-H diagram because the $A$-plane curves for SN-PD-EH already include intersections of Hopf curve endpoints (TakensBogdanov and double-negative-one points) with other curves when projected to the $C$ plane. Our count was 380 distinct regions: 26 on the real $A$ axis, 177 in the upper half $A$ plane and 177 conjugate regions in the lower half $A$ plane. The result is diaplayed in Fig. 8. For comparison with Fig. 7, we have included the same 33 marked points on the real $A$ axis in Fig. 8. Several of the $A$ values that corresponded to distinct equivalence classes in Fig. 7 are in the same equivalence classes in Fig. 8. See, for example, $A$ values numbered 16-18, 20-21, and $22-23$ in Fig. 8 b. The corresponding $C$-plane bifurcation diagrams are the same as pictured in Fig. 7c. We merely ignore the cyan portions of the EH curve. For example, from $C$ plane 17 to 18, the left-hand (green) saddle-node cusp pushes through a cyan portion of the EH curve. Numbers 16 and 17 appear in the same equivalence class in Fig. 8b, but were in distinct equivalence classes in $7 \mathrm{~b}$.

As was the case for Fig. 7, we have tried to make the bifurcation diagram in Fig. 8 as complete as possible but make no claims as to its completeness. We also have not made any attempt to fully explain the diagram to the reader. We have only attempted to communicate the type of information one might obtain from such a bifurcation diagram.

\subsection{The local saddle-node unfolding.}

There are many points in the SN-PD-EH and SN-PD-H $A$-plane bifurcation diagrams that would be interesting to investigate in more detail. We treat only one such point, the local saddle-node point, here. More specifically, we investigate the bifurcations of the SN-PD-EH (resp. SN-PD-H) curves near $(z, C, A)=(0,0,0)$. This point is of interest because at $A=0,(z, C)=(0,0)$ is the cusp of the main cardioid of the Mandelbrot set (point $H^{0 / 1}$ in Fig. 1a). This point is often called the saddle-node point of the Mandelbrot set. By considering the fixed-point bifurcations for $A$ values near zero, we are investigating what happens to these sets as a complex analytic family $(A=0)$ is perturbed with a non-complex-analytic term. In Peckham [1998] we showed that for $A \neq 0$ the saddle-node set was a topological circle in the $(z, C)$ variables. It projected to a three-cusped triangular shape in the $C$ plane. We did not consider in that paper the $\mathrm{EH}$ curve or its interaction with the saddle-node triangle. The period-doublings play no role in this bifurcation since there are none in the neighborhood of $(z, C, A)=(0,0,0)$. (There are period-doublings in the neighborhood of $(z, C, A)=(-1,-1,0)$.)

The bifurcation diagram, an enlargement of Fig. 7 near $A=0$, is shown in Fig.9. The numbering of the regions does not match that of Fig.7. 
Place Figure 9 near here.

As one approaches $A=0$ along any one of the six bifurcation curves that runs through the origin, the phase $(z)$ and primary parameter $(C)$ value of any point involved in the bifurcation approaches $(z, C)=(0,0)$. These six curves therefore are the bifurcation curves for a two-real-parameter $(A)$ local unfolding of the saddle-node point $\left(z+z^{2}+C\right.$ near $\left.(z, C)=(0,0)\right)$. Because the $A$-plane bifurcation diagram has more than one bifurcation curve passing through the origin it must be of codimension at least two in the space of local two-realparameter families of maps of the plane. We conjecture that the codimension is exactly two. In contrast, when considering either the saddle-node locus or the Hopf locus by itself, the saddle-node bifurcation appears to be of real codimension one in the sense that all equivalence classes of local primary parameter plane bifurcation diagrams for nearby auxiliary parameter values are present in a typical (transversal) one-auxiliary-parameter family.

The bifurcation sequences are best understood via Fig. 9. For the details of the transition between C-planes 1-2-3-4 look at the blowup of C-plane number 2 in the inset. Label the two branches of Hopf points as upper and lower according to the Takens-Bogdanov point from which they come. As the A value progresses from locations 1 through 4 in Fig. 9a, the lower branch of the Hopf bifurcations (the closer to vertical in the insert of Fig.9b) moves from right to left. Bifurcations occur when this lower Hopf branch crosses through the three points indicated by the arrows: the saddle-node cusp point, the point where the upper Hopf branch intersects the right-hand side of the saddle-node triangle (labelled H-SN projection intersection), and the Takens-Bogdanov point. We note that the bifurcation diagram is the same whether we consider extended Hopf or Hopf points. In all cases except along the EH cusp curve, this is relatively obvious since the $\mathrm{EH}$ point in question is always a true Hopf point. The EH cusp curve is more subtle. This curve was included in the EH-only bifurcation diagram of Fig. 5, but not in the H-only bifurcation diagram of Fig. 6 since on either side of the bifurcation curve, the corresponding Hopf curve was merely a line segment with an endpoint. Here, however, we are considering all three sets together. In particular, the intersection of the (lower) Hopf segment with the lower left side of the period doubling triangle changes orientation as A passes through a value for which the EH curve has a cusp: the lower Hopf branch approaches the lower Takens-Bogdanov point tangent to the saddle-node curve from below in C-plane 5, but from above in C-plane 6. On the dividing line, the Hopf curves approaches transverse to the saddle-node curve (where the full EH curve forms a cusp). Between C-planes 6 and 7 the saddle-node triangle rotates roughly one-sixth of a full rotation in the clockwise direction. In the process, the TB point on the right in $C$-plane 6 passes through the saddle-node cusp and ends up on the lower left leg of the triangle in $C$-plane 7 . 


\section{Classification of codmension-one bifurcations}

In the previous section we discussed bifurcation curves in the auxiliary $A$ plane that separated equivalence classes of $C$-plane bifurcation diagrams. These curves are all codimension-one in the auxiliary parameter plane. We now classify these bifurcation curves. More generally, we are classifying bifurcations for families with two real primary parameters. The statements below assume two auxiliary parameters although analogous statements hold for any number of auxiliary parameters. We divide the classification into two primary groups: those that involve bifurcation points which are local in the six-dimensional $(z, C, A)$ space, and those that are not. Actually, the only variable which is allowed to be "nonlocal" is the phase variable $z$. This is because we are considering local features of the primary $(C)$ plane that change as the auxiliary parameter $(A)$ is varied (by an arbitrarily small amount). We treat the nonlocal group first.

\subsection{Bifurcations which are nonlocal in $z$}

Conjecture 3.1 Consider a region of auxiliary parameter space where the specified primary plane (traditional codimension-one) bifurcation curves are in fact curves - immersed one-manifolds in the full six-dimensional space, either with or without endpoints. The only nonlocal codimension-one bifurcations in the space of two-real-parameter families of maps of the plane are of the following three types:

\section{Tangent projection of two traditional codimension-one curves.}

2. Triple projection intersection of traditional codimension-one curves.

3. Projection intersection of a traditional codimension-one curve with a distinguished point on a traditional codimension-one curve.

Note that the assumptions of our hypothesis are satisfied for the fixed-point SN, $\mathrm{PD}, \mathrm{EH}$, and $\mathrm{H}$ curves except at $A=0$, where the $\mathrm{SN}$ and PD points degenerate to a point. For $A \neq 0$ the first three are closed curves and the $\mathrm{H}$ set is a pair of line segments.

\subsubsection{Tangent projection of two traditional codimension-one curves.}

Two bifurcation curves which are traditionally codimension-one (saddle-nodes, period-doublings, and Hopfs, for example) live in the four-dimensional $(z, C)$ space for fixed auxiliary parameter values. These curves, or different parts of the same curve, can have points which have distinct $z$ values but a common $C$ value. When the curves are also tangent in their $C$ plane projection, the local topology of their intersection in the $C$ plane is unstable with respect to perturbation by the auxiliary parmeter: the curves can either push through each other or separate from each other. Such bifurcations include those labelled in 
this paper as PD-PD, EH-EH, H-H, SN-EH, SN-H, PD-EH, PD-H and SN-PD tangent projection intersections.

\subsubsection{Triple projection intersection of codimension-one curves}

If three points are on traditional codimension-one curves and have distinct $z$ values but a common $C$ value, then the local topology of the projection to the $C$ plane can change as the auxiliary parameter is varied. Such bifurcations in include those labelled in this paper as EH-EH-EH, EH-EH-SN, H-H-SN, EHEH-PD, H-H-PD, PD-PD-EH and PD-PD-H projection intersections.

\subsubsection{Projection intersection of a traditional codimension-one curve with a distinguished point on a traditional codimension-one curve}

If two points, one a distinguished point of a specified bifurcation curve (a traditional codimension-two point) and the other on a specified bifurcation curve, have distinct $z$ values but a common $C$ value, then the topology of their projection to the $C$ plane can change as the auxiliary parameter is varied. The distinguished point can either be interior to the specified bifurcation curve, as is a saddle-node cusp on a saddle-node curve, or a ONO point on either a saddlenode or period doubling curve, or it can be an endpoint of a specified bifurcation curve, as is a TB point or a DNO point on a Hopf curve. Such bifurcations include those labelled in this paper as TB-EH, SNcusp-EH, SNcusp-H, DNO-PD, DNO-EH, DNO-H, ONO-PD, SNcusp-PD, DNO-SN, TB-PD, ONO-EH and ONO-H projection intersections.

\subsection{Bifurcations which are local in $\mathcal{R}^{6}$}

The codimension-one $A$-plane bifurcation curves which in this paper involve points which are local for $(z, C, A) \in \mathcal{R}^{6}$ all correspond to traditional local codimension-three bifurcations. There are five such bifurcations present in our study, each related to a traditional codimension-two point with an additional degeneracy. The codimension-two points are either Takens-Bogdanov points or one-negative-one points, so we first recall the model normal form unfoldings for these two traditional codimension-two points. We note that although the universal unfolding and corresponding dynamics of a Takens-Bogdanov for a differential equation has been established ([Ta, 1974], [Bo 1976]), results for the TB and ONO points for maps are less complete. This is not an issue for us, however, since we are only considering fixed points and bifurcation sets but not the full dynamics of the maps. 
Takens-Bogdanov points. A model normal form unfolding of a TakensBogdanov point is

$$
(x, y) \rightarrow\left(x+y, \epsilon_{1}+\epsilon_{2} y+b_{20} x^{2}+b_{11} x y\right) . \quad\left(T B_{\text {normal }}\right)
$$

A generic two-parameter family of maps of the plane which has a fixed point with a double one eigenvalue but only a one-dimensional corresponding eigenspace can be put into this form up through its quadratic terms by standard local changes of variables (phase variables and parameter variables). The unfolding parameters are $\epsilon_{1}$ and $\epsilon_{2}$. The higher order terms have been dropped. The unfoldings are divided into cases, depending on the signs of $b_{20}$ and $b_{11}-2 b_{20}$. These two quantities being nonzero are nondegeneracy conditions.

One-negative-one points. The model normal form unfolding of a one-negativeone point is

$$
(x, y) \rightarrow\left(\epsilon_{1}+x+a_{20} x^{2}+a_{02} y^{2}, \epsilon_{2} y-y+b_{11} x y+b_{03} y^{3}\right) . \quad\left(\text { ONO } O_{\text {normal }}\right)
$$

A generic two-parameter family of maps of the plane which has a fixed point with eigenvalues of one and negative one can be put into this form by making standard local changes of variables and dropping higher order terms. The unfoldings are divided into cases depending on the signs of $b_{11}$ and $a_{20}$. These two coefficients being nonzero are nondegeneracy conditions.

We are now able to state, in the following proposition, the relationship between the five codimension-one auxiliary parameter bifurcations and their corresponding traditional codimension-one bifurcations.

Proposition 3.2 The following auxiliary parameter space codimension-one bifurcation loci correspond to the respective traditional codimension-three bifurcations:

$\begin{array}{lll} & \text { auxiliary parameter space } & \text { Traditional codimension-three } \\ & \text { codimension-one bifurcation } & \text { bifurcation (in normal form) } \\ \text { 1. } & \text { EHcusp } & \text { T-B point with } b_{11}-2 b_{20}=0 \\ \text { 2. } & \text { EH-SNcusp } & \text { T-B point with } b_{20}=0 \\ \text { 3. } & \text { PDcusp } & \text { ONO point with } b_{11}=0 \\ \text { 4. } & \text { PD-SNcusp } & \text { ONO point with } a_{20}=0 \\ \text { 5. } & \text { Double ONO } & \text { ONO point with a parametric degeneracy* }\end{array}$

*Explained further below

Proof (Sketch.) The most interesting observation in this proof is the fact that cusps on Extended Hopf curves or on period-doubling curves are possible only when these points also have an eigenvalue of 1 . The rest of the details are relatively straightforward computations, albeit not trivial.

1. We note from eq. ( $\left.E H_{\text {par }}\right)$ in sec 2.3 that for $A$ fixed, the Extended Hopf curve in $\mathcal{R}^{4}((z, C)$-space) is a smooth curve parametrized by 
$\theta_{H} \mapsto\left(-\frac{1}{2}+\frac{\sqrt{|A|^{2}+1}}{2} e^{i \theta_{H}},-\left(-\frac{1}{2}+\frac{\sqrt{|A|^{2}+1}}{2} e^{i \theta_{H}}\right)^{2}-A\left(-\frac{1}{2}+\frac{\sqrt{|A|^{2}+1}}{2} e^{-i \theta_{H}}\right)\right)$.

(The derivative with respect to $\theta_{H}$ in the $z$-component is nonzero, so the curve is smooth even if the $C$-component has zero derivative.) Moreover, this curve lies on the smooth surface of fixed points given by $C+z^{2}+A \bar{z}=0$.

Thus, we have a smooth curve lying on a smooth surface in $\mathcal{R}^{4}$. Moreover, the projection of this surface to the $C$-plane is a local diffeomorphism away from the branch points, or the saddle-node points. It follows that away from the saddle-node curve the projection of the Extended Hopf curve into the $C$ plane is non-singular. Thus singular points of the Extended Hopf curve can only occur when the Extended Hopf curve intersects the saddle-node curve. Being on both the Extended Hopf and saddle-node curves forces both eigenvalues to be one. Such a point is (generically in two-parameter families) a nondegenerate Takens-Bogdanov point.

Further, at a nondegenerate Takens-Bogdanov point, the Extended Hopf bifurcation curve and the saddle-node curve both are smooth curves whose projections to the $\left(\epsilon_{1}, \epsilon_{2}\right)$ parameter plane intersect at the origin with quadratic tangency. More specifically, from the Takens-Bogdanov normal form of eq. ( $T B_{\text {normal }}$ ), one can calculate that if the quantities $b_{20}$ and $b_{11}-2 b_{20}$ are both nonzero, the saddle-node curve projects to the $\epsilon_{2}$ axis in the $\left(\epsilon_{1}, \epsilon_{2}\right)$ parameter plane, and the Extended Hopf curve projects to the parameter plane as the parabola $\left(2 b_{20}-b_{11}\right)^{2} \epsilon_{1}=-b_{20} \epsilon_{2}^{2}$. Thus, a cusp on the Extended Hopf curve can only occur when the Takens-Bogdanov point has an additional degeneracy. That the cusp corresponds to the degeneracy $b_{11}-2 b_{20}=0$ rather than $b_{20}=0$ is a calculation.

Instead of performing that calculation here, we provide Fig. 10. The Fig. 10 sequence was computed using eq. (T $\left.B_{\text {normal }}\right)$ with $b_{20}=1, b_{11}$ taking on the three values $1,2,3$, and some higher order terms $\left(x^{3}+x^{4}\right)$, chosen to make the family generic when the nondegeneracy condition $b_{11}-2 b_{20} \neq 0$ fails, added to the second component.

Place Figure 10 near here.

In all three parts of Fig. 10, the fixed-point surface, the green saddle-node curve along the surface "folds," and the projection of the saddle-node curve to the $\left(\epsilon_{1}, \epsilon_{2}\right)$ parameter plane are similar. The projection of the Extended Hopf curve to the parameter plane, however, changes. When $b_{11}=1,2 b_{20}-b_{11}>0$ and the Extended Hopf curve (including both the red branch labelled $\mathrm{H}$ and the cyan branch labelled EH) is tangent to the saddle-node curve when projected to the parameter plane, shown at the bottom of the box. When $b_{11}=2$, $2 b_{20}-b_{11}=0$ the projected Extended Hopf curve forms a cusp where it intersects 
the saddle-node curve. When $b_{11}=3,2 b_{20}-b_{11}<0$ the projected Extended Hopf curve is again tangent to the saddle-node curve, but the true Hopf and Extended Hopf branches have switched sides.

2. This correspondence will be relatively obvious to those who are familiar with the two-parameter unfolding of the cusp bifurcation for maps of the line: $x \mapsto x+\epsilon_{1}+\epsilon_{2} x+x^{3}$. It is straightforward to calculate from eq. ( $\left.T B_{\text {normal }}\right)$ that the saddle-node curve can only have a cusp if $b_{20}=0$.

3. This proof is similar to the proof of part 1. For the same reasons, a cusp on a period-doubling curve must also have an eigenvalue of one. Consequently, it must be a ONO point. The PD curve and SN curve typically have a tangent intersection in the $\left(\epsilon_{1}, \epsilon_{2}\right)$ parameter plane, but when $b_{11}=0$ in the perioddoubling curve has a cusp.

4. This proof is similar to the proof of 2. Details are omitted.

5. This (traditional) codimension-three bifurcation is somewhat different from the other four. Two degeneracies are the one and negative one eigenvalues. The third is not a degeneracy in the normal form at the bifurcation point, but rather a degeneracy in the unfolding. It would be modelled by a three-parameter family obtained from eq. $\left(O N O_{\text {normal }}\right)$ by replacing $\epsilon_{2}$ with $\epsilon_{3}+\epsilon_{2}^{2}$. The third (auxiliary) parameter $\epsilon_{3}$ in the model corresponds to $|A|$ in the paper's model. When $\epsilon_{3}$ is fixed negative $(|A|$ is fixed greater than one), the resulting twoparameter family has two ONO points; when $\epsilon_{3}$ is fixed positive $(|A|$ is fixed less than one), the resulting two-parameter family has no ONO points.

Explicit Calculations. The above proposition, especially for cases 1 and 3, changes the task of the explicit computation of the bifurcations loci for each of the 5 cases listed above from nearly impossible to merely tedious. For example, to compute the EHcusp points, we know from the proposition that they occur when we have a Takens-Bogdanov point with a higher order degeneracy. So we start with the parametrization for a Takens-Bogdanov point, convert it to normal form, and set the combination $b_{11}-2 b_{20}=0$. We include only the results but not the intermediate calculations here. Note in 1,3 , and 4 , the $C$ values are not explicitly written out, but are given by the fixed-point condition $C=-z^{2}-A \bar{z}$.

Parametric versions are, respectively:

1.

$$
\theta_{A} \mapsto(z, C, A)=\left(-\frac{\left(\sin \left(\theta_{A}\right)-1\right) i}{2 \cos \left(\theta_{A}\right)}, C, \frac{\sin \left(\theta_{A}\right)-1}{\cos \left(\theta_{A}\right)} e^{i \theta_{A}}\right), \theta_{A} \in \mathcal{S} \quad\left(\text { EH }_{\text {cusp }} p_{\text {par }}\right)
$$

(which is equivalent to the nonparametric cubic curve:

$$
\left.A_{1}+\left(2+A_{1}\right)\left(A_{1}^{2}+A_{2}^{2}\right)=0\right)
$$


2 .

$$
y \mapsto(z, C, A)=\left(y i, 3 y^{2},-2 y i\right), y \in \mathcal{R} \backslash\{0\} \quad\left((E H-S N c u s p)_{\text {par }}\right)
$$

3 .

$$
A_{2} \mapsto(z, C, A)=\left(-\frac{1}{2}+i y, C,-1-i 2 y\right), y \in \mathcal{R} . \quad\left(P D c u s p_{p a r}\right)
$$

4.

$$
\begin{array}{r}
\theta_{A} \mapsto(z, C, A)=\left(-\frac{1}{2}+y i, C,-\left(\frac{1}{4}+y^{2}\right)^{\frac{1}{2}} e^{3 i \arctan (2 y)}\right), y \in \mathcal{R} . \\
\left((P D-S N c u s p)_{p a r}\right)
\end{array}
$$

5.

$$
\theta_{A} \mapsto(z, C, A)=\left(-\frac{1}{2},-\frac{1}{4}+\frac{1}{2} e^{i \theta_{A}}, e^{i \theta_{A}}\right), \theta_{A} \in \mathcal{S} . \quad\left((\text { double }-O N O)_{p a r}\right)
$$

\section{Continuation Comments}

The five bifurcation curves whose explicit formulas were just presented above in sec 3.2 were computed using those formulas. All other bifurcation curves were computed via numerical continuation using the dynamical systems software package To Be Continued ... [Peckham, 1988-present]. Previously developed software allowed computation of traditional codimension-one $C$-plane curves such as saddle-nodes, period-doublings and Hopfs. New routines were written to handle the bifurcation curves in the $A$ plane.

For example, for the triple intersections of the extended Hopf locus, we solved for $\left(\theta_{1}, \theta_{2}, \theta_{3}, A\right) \in \mathcal{S} \times \mathcal{S} \times \mathcal{S} \times \mathcal{R}^{2}$ :

$$
C\left(A, \theta_{1}\right)=C\left(A, \theta_{2}\right), C\left(A, \theta_{2}\right)=C\left(A, \theta_{3}\right)
$$

where $C(A, \theta)$ was explicitly defined by the middle coordinate of eqn. $\left(E H_{p a r}\right)$ in sec 2.3. This is equivalent to four real equations in five real variables, resulting in a curve. Projecting to the $A$ plane then gave us our $A$-plane bifurcation curves.

Cheating for tangent intersections. The tangent intersections of two bifurcation curves projected to the $C$-plane, written parametrically as $\gamma_{1}(A, t)$ and $\gamma_{2}(A, s)$, where $\gamma_{i}: \mathcal{R}^{2} \times \mathcal{R} \rightarrow \mathcal{R}^{2}$ (as in the middle component of eqs. $\left.\left(S N_{\text {par }}\right),\left(P D_{\text {par }}\right),\left(E H_{\text {par }}\right)\right)$, were computed using the following observation. The direct tangency conditions are:

$$
\gamma_{1}(A, t)=\gamma_{2}(A, s), \frac{d}{d t} \gamma_{1}(A, t)=\lambda \frac{d}{d s} \gamma_{2}(A, s)
$$

where $\lambda$ is any nonzero real number. They are satisfied precisely at the points where the related family of map of the plane:

$$
G_{A}(t, s) \equiv\left(t+\pi_{1}\left(\gamma_{1}(A, t)-\gamma_{2}(A, s), s+\gamma_{2}(A, t)-\gamma_{2}(A, s)\right.\right.
$$


has a fixed point with eigenvalue 1 . That is, $G_{A}$ has a saddle-node bifurcation. This meant no new software had to be created for these bifurcations. We merely entered the appropriate related map " $\mathrm{G}$ " and found its saddle-node bifurcation points. We note that the cusp points (saddle-nodes with a higher order degeneracy) for the related map correspond to curves which intersect not only with matching tangent vectors but also with matching curvature. See $C$ plane 7 in Fig.6, for example.

\section{Summary}

In this paper we have studied the evolution of parameter plane bifurcation diagrams as auxiliary parameters are varied. In retrospect, it is not so surprising that the bifurcation analysis is so complicated since the family has four parameters, especially because we did not restrict the parameters to some local neighborhood. Nevertheless, we feel that the evolution of planar bifurcation diagrams is of enough general interest to warrant its study. Further, we believe the particular family we studied, $z \mapsto z+z^{2}+C+A \bar{z}$ - being a familiar complex quadratic map at $A=0$ and being perturbed by the simplest non-complexanalytic term, $A \bar{z}$ - is a reasonable prototype for studying parameter plane evolution. Thus we have worked to obtain complete bifurcation diagrams for this example. We conjecture that the $A$-plane bifurcation diagrams of figures 4 through 9 are complete, with respect to the bifurcation loci specified for each figure.

With this study, global in the parameters $C$ and $A$, as a guide, we hope to return to certain points in our $A$-plane bifurcation diagrams which seem to require further study. Neighborhoods of $A=0$ are of particular interest since they represent bifurcation diagrams of maps which are close to complex analytic. For example, we would like to be able to verify our conjecture that the saddlenode point (near $(z, C, A)=(0,0,0))$ is codimension one with respect to saddlenode or Extended Hopf points separately, but codimension-two with respect to both loci together. Similar studies near bulb contact points of the Mandlebrot set (also near $A=0$ but not near $(z, A)=(0,0)$ ) require further attention. They will require looking at additional bifurcation loci beyond the fixed-point loci considered in this paper. For example, for the period doubling bifurcation, near $(z, C, A)=(-1,-1,0)$, at least period-two saddle-node bifurcations should be added to the mix.

Note that while the focus on bifurcation sets allows us to more easily deal with whole families of maps, it also necessarily ignores many interesting questions about the dynamics of these noninvertible maps of the plane. For example, a complete description of the dynamics would necessarily involve the study of the critical set and its images, such as in Nien [1998]. There is a need for much additional research in this area.

Ideally, we hope this work will encourage and complement further work on 
both the bifurcations and the dynamics of noninvertible maps of the plane.

\section{Acknowledgements}

Our idea of studying bifurcations only with respect to a specified collections of bifurcation loci is similar in spirit to the idea of R. P. McGehee and C-H Nien [Nien, 1997] to study the equivalence classes of fixed points of noninvertible planar maps with a one eigenvalue and a zero eigenvalue "with respect to certain bifurcation phenomena." 


\section{$7 \quad$ References}

Bielefeld B., Sutherland S., Tangerman F., Veerman J.J.P. [1993], "Dynamics of Certain Nonconformal Degree-Two Maps of the Plane," Experimental Mathematics 2 No. 4, 281-300.

Bogdanov R.I. [1976], "Versal deformation of a singularity of a vector field in the plane in the case of zero eigenvalues," Trudy Seminara Imeni I. G. Petrovskogo, Vol. 2, 23-36, 1976. English translation: Selecta Math. Sovietica, Vol. 1, No. 4, 389-421, 1981.

Carleson L. and Gamelin T.W., [1993], Complex Dynamics, Springer-Verlag, New York.

Drexler J. [1966], "A Nonanalytic Perturbation of the Complex Quadratic Family of Maps," Master's Project University of Minnesota, Duluth, Technical Report 96-5.

Golubitsky M. and Schaeffer D. [1985], Singularities and Groups in Bifurcation Theory, Volume I, Applied Mathematical Sciences, 51, Springer Verlag, New York.

Nien C-H [1998], "The Dynamics of Planar Quadratic Maps with Nonempty Bounded Critical Set," International Journal of Bifurcations and Chaos, Vol. 8, No. 1.

Nien C-H [1997], "The investigation of a saddle-node bifurcation with a zero eigenvalue - includes examle of non-analyticity," Ph. D. Thesis, University of Minnesota.

Peckham, B.B. [1988-98], To Be Continued ..., a continuation software package for discrete dynamical systems (continually under development).

Peckham B.B. [1998], "Real Perturbation of Complex Analytic Families: Points to Regions," International Journal of Bifurcations and Chaos, Vol. 8, No. 1, 73-93, 1998.

Takens F. [1974], "Forced oscillations and bifurcations," Applications of Global Analysis, Communications of the Mathematical Institute Rijksuniversiteit Utrecht, Vol. 3, 1-59. 


\section{Figures}

Fig. 7 and Fig. 8 labels. The $A$-space curves that appear for the above sets of loci have the following common labels. We abbreviate saddle-node as SN, period-doubling as PD, Extended Hopf as EH, Hopf as H, Takens-Bogdanov point as TB, double negative one point as $\mathrm{DNO}$, and a one, negative one point as ONO.

a PD cusp

b PD-PD tangent projection intersection

c EH cusp

d EH-EH tangent projection intersection

e EH-EH-EH projection intersection

f SN-EH tangent projection intersection

g SNcusp-EH intersection

$\mathrm{h}$ TB-EH projection intersection

i EH-EH-SN projection intersection

j SNcusp-EH projection intersection

$\mathrm{k}$ DNO-PD projection intersection

1 PD-EH tangent projection intersection

$m$ DNO-EH projection intersection

n EH-EH-PD projection intersection

o PD-EH tangent projection intersection

p SN-PD tangent projection intersection

q SNcusp-PD intersection

r ONO-PD projection intersection

s SNcusp-PD projection intersection

t double ONO points (the two ONO points have coalesced)

u DNO-SN projection intersection

$\mathrm{v}$ TB-PD projection intersection

$\mathrm{w}$ ONO-EH projection intersection

$\mathrm{x}$ PD-PD-EH projection intersection 

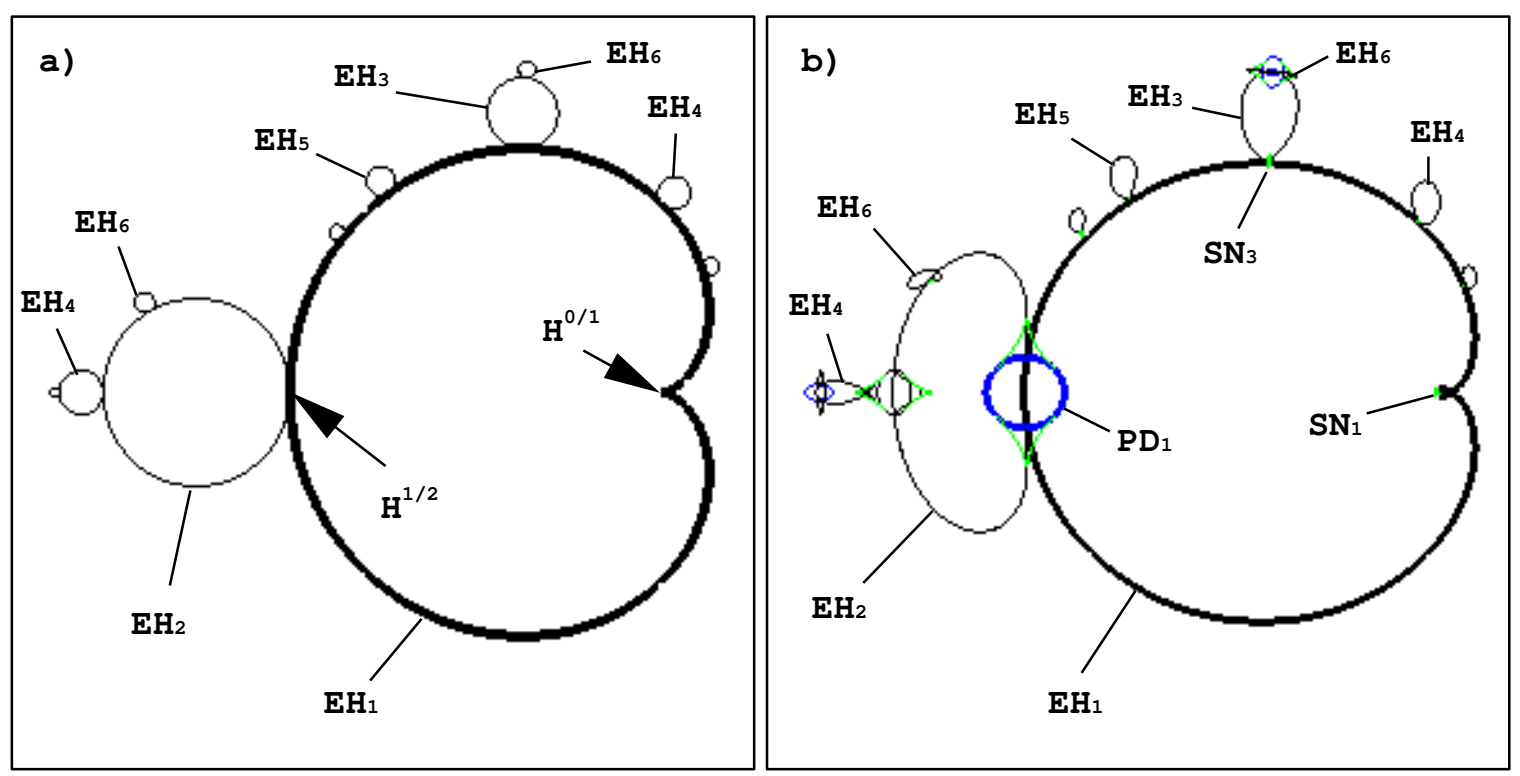

Figure 1: For $Q_{(\tilde{C}, A)}(z)=z^{2}+\tilde{C}+A \bar{z}$ : a) Some numerically computed bulb boundaries of the Mandlebrot set $(A=0)$. b) Numerically computed $\tilde{C}$ plane bifurcation diagrams for $A=-0.1$. Period-doubling curves are in blue; saddle-node curves are in green. Curves of interest to the current paper are wider: $E H_{1}, S N_{1}, P D_{1}$. Reprinted from Peckham [1998]. 


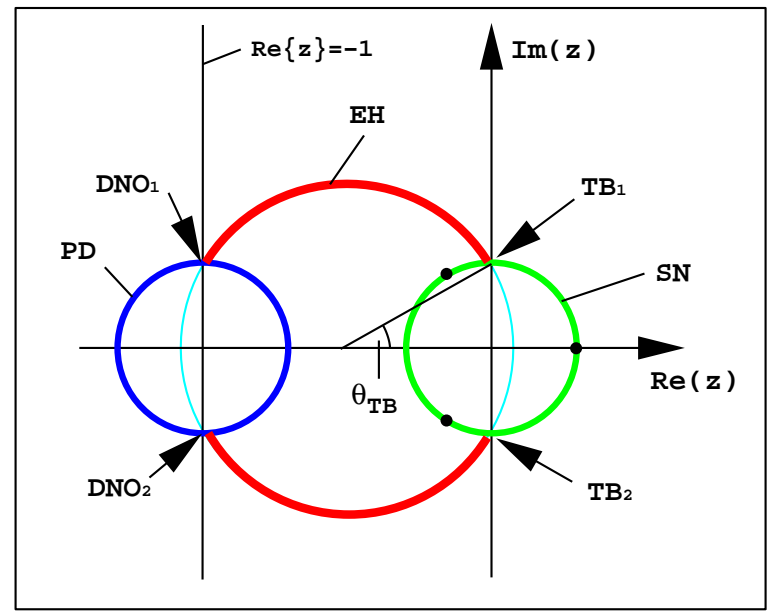

Figure 2: Projection of the three loci to the phase plane. $(0<|A|<1)$. Symbols: SN: saddle-node, PD: period-doubling, EH: extended Hopf (true Hopf points are the bold red part of the EH circle), TB: Takens-Bogdanov point (double one eigenvalues), DNO: double negative one eigenvalue point, ONO: eigenvalues of one and negative one (present only if $|A| \geq 1$ ), $\theta_{T B}$ : angle of first quadrant TB point. The radii of the PD and SN circles are both $\frac{|A|}{2}$; the radius of the EH circle is $\frac{\left(|A|^{2}+1\right)^{1 / 2}}{2}$. The three marked points on the saddle-node curve are the cusps; their location on the saddle-node circle was determined by assuming $\arg (A)=0$.

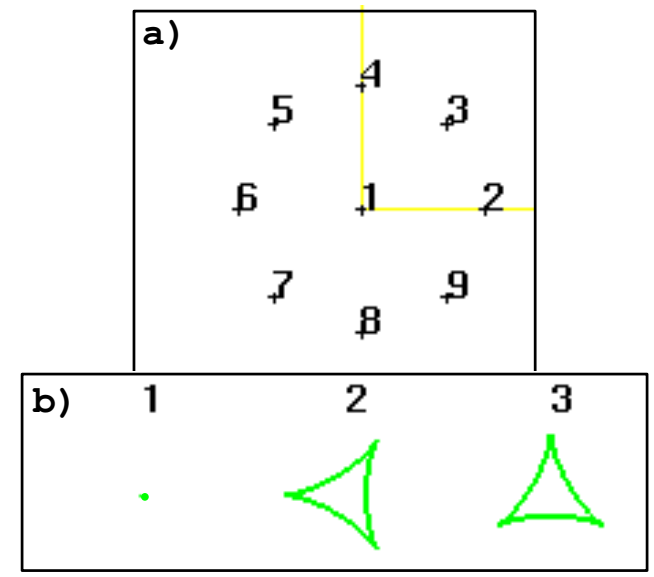

Figure 3: Fixed-point saddle-nodes only. a) The $A$-value at which the saddle-node locus bifurcates. The origin (number 1 ) is the only bifurcation value. Numbers 2 through 10 (arbitrarily chosen on the unit circle), as well as any $A$ value except the origin, are in the same equivalence class since only fixed-point saddle-node curves are considered. b) Some of the orresponding saddle-node curves in the $\mathrm{C}$ plane. 


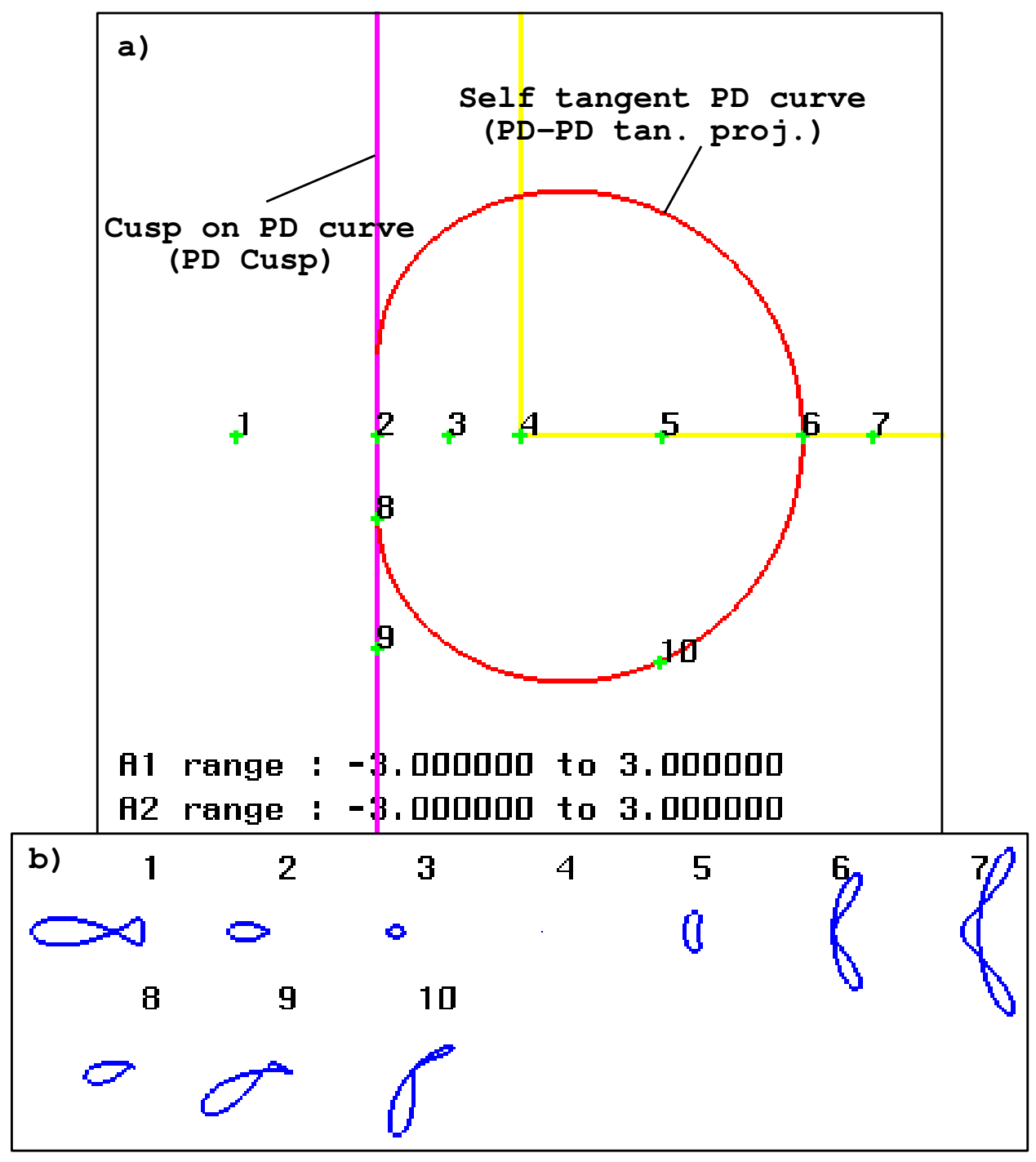

Figure 4: Fixed-point period-doublings only. a) The $A$-values at which the perioddoubling locus bifurcates. b) Corresponding period-doubling curves in the C plane 